\title{
ESTIMULAÇÃO CEREBRAL NÃO INVASIVA EXCITATÓRIA SOBRE A ATENÇÃO DE ADULTOS COM SINTOMAS DO TRANSTORNO DE DÉFICIT DE ATENÇÃO E HIPERATIVIDADE
}

\author{
Excitatory non-invasive brain stimulation over attention of adults with symptoms of \\ Attention-deficit hyperactivity disorder \\ Tarcisio Dutra, Águida Foerster, Adriana Baltar, María Lucia Gurgel, Katia Monte-Silva \\ Federal University of Pernambuco, Brasil \\ (Rec.: julio de 2017 - Acept.: octubre de 2017)
}

\begin{abstract}
Resumo
Objetivos: avaliar a atenção de adultos com sintomas de transtorno de déficit de atenção e hiperatividade (TDAH), quando submetidos a técnicas de estimulação cerebral não invasiva (ECNI) excitatória. Métodos: nesse estudo crossover 20 voluntários foram submetidos a uma sessão real e uma sham de Estimulação Magnética Transcraniana Repetitiva (EMTr) ou Estimulação Transcraniana por Corrente Contínua (ETCC). A EMTr (10Hz) foi aplicada no córtex dorsolateral esquerdo. A ETCC foi aplicada colocando-se o ânodo no mesmo local e cátodo na região supraorbital contralateral (1mA e 20 minutos). A avaliação foi realizada através de testes neuropsicológicos, considerando os desfechos: atenção focada e sustentada; amplitude atencional, manipulação mental e resistência à interferência. Resultados: observou-se que a ECNI excitatória exerceu efeito distinto entre os grupos, prejudicando o desempenho atencional dos adultos saudáveis e beneficiando os pacientes com TDAH. Destaca-se que em relação à estimulação sham o grupo controle aumentou o desempenho atencional de forma significativa, possivelmente decorrente do efeito de aprendizagem, enquanto o grupo com TDAH só obteve aumento no desempenho após a aplicação da estimulação real. Conclusão: os achados ratificam a compreensão que a ECNI excitatória pode contribuir para melhora cognitiva em adultos com TDAH e sugerem que processo inverso pode acontecer em relação aos saudáveis.
\end{abstract}

Descritores: Transtorno de Déficit de Atenção e Hiperatividade; Adulto; Neuropsicologia; Estimulação Elétrica

\begin{abstract}
Purpose: evaluate the attentional performance of adults with symptoms of Attention-deficit hyperactivity disorder (ADHD) when submitted to excitatory non-invasive brain stimulation (NIBS). Methods: a crossover design were performed with 20 volunteers underwent to a real session and sham session using repetitive Transcranial magnetic stimulation (rTMS) or Transcranial direct current stimulation (tDCS). The rTMS (10Hz) was applied over left dorsolateral cortex. The tDCS was applied in the same place and the cathode over the contralateral orbital region ( $1 \mathrm{~mA}$ and $20 \mathrm{mi}-$ nutes). The evaluation was done trought the neuropsycologhical tests regarding the following outcomes: focused and sustained attention; Attentional amplitude, mental manipulation (and resistance to interference. Results: it was observed that excitatory stimulation induces a distinct effect between groups, harming the atentional performance of health adults and improving the performance of the subjects with Attention-deficit hyperactivity disorder. It is important to highlight that the sham stimulation in control group increase the attentional performance, possible due the learning effect. In the other hand, the group with Attention-deficit hyperactivity disorder symptoms only has an increase in performance after the real stimulation. Conclusion: this findings help in urderstading of excitatory NIBS could contribute to improve the cognitive performance in adults with ADHD and the opposite can happen with healthy subjects.
\end{abstract}

Keywords: Attention Deficit Disorder with Hyperactivity; Adult;Neuropsychology; Eletric Stimulation

\footnotetext{
* Correspondencia: Tarcisio Dutra. Federal University of Penambuco, Brasil.
}

Email: tgdutra@yahoo.com 


\section{Introdução}

O Transtorno de déficit de atenção e hiperatividade (TDAH) é uma condição neuropsiquiátrica que emerge durante a infância e, muitas vezes, persiste na vida adulta, apresentando como principais sintomas a desatenção, a hiperatividade e a impulsividade 1. A prevalência do transtorno na população adulta é estimada entre 3,4\% e 4,4\% (Woltering, Liu, Rokeach \& Tannock., 2013). Nestes, a hiperatividade é muitas vezes evidenciada como uma sensação subjetiva de inquietude interna (Missonnier et al., 2013). Nos adultos, além dos sintomas característicos do transtorno, os indivíduos comumente apresentam instabilidade emocional, temperamento explosivo e déficits neuropsicológicos nos domínios da atenção, inibição comportamental e memória de trabalho (Hervey, Epstein \& Curry, 2004). Tais aspectos interferem no âmbito profissional, acadêmico, comportamental, social e afetivo do pacientes, além de prejudicar a qualidade de vida (Woltering et al., 2013).

Algumas áreas cerebrais, tais como os córtices pré-frontais direito e esquerdo, núcleo caudado, globo pálido (Casey et al., 1997), córtex cingulado anterior (Bush et al., 1999) e a área rostral do corpo caloso (Giedd, 1994) têm sido relacionadas aos déficits neuropsicológicos no TDAH. Estudos que utilizam instrumentos de neuroimagem têm revelado que a atividade cerebral em pacientes com TDAH é reduzida na região préfrontal dos hemisférios cerebrais. O córtex pré-frontal possui importantes funções cognitivas, tais como inibição comportamental, atenção seletiva, autocontrole, criação de estratégias para resolver problemas e planejamento ações, todas achamse gravemente perturbadas nos portadores de TDAH (Benczik, 2000). Além disso, sabe-se que o sistema dopaminérgico parece estar afetado no TDAH (Ernst et al., 1999). Portanto, um tratamento que pudesse aumentar a concentração de dopamina no córtex pré-frontal poderia ter um efeito positivo no quadro clínico de pacientes portadores do TDAH. De fato, os estudos farmacológicos que utilizaram metilfenidato no tratamento do TDAH mostraram que o medicamento aumenta a quantidade de dopamina no cérebro, reduzindo assim os sintomas (Faraone et al., 2004).

Neste contexto, as técnicas de estimulação cerebrais não-invasivas parecem ser um método de tratamento em potencial para o TDAH. Strafella, Paus, Barret \& Dagher et al., (2001) mostraram que a estimulação magnética transcraniana repetitiva (EMTr) aplicada sobre o córtex pré-frontal induziu a liberação de dopamina endógena. Strafella e Cho também forneceram evidência da modulação do sistema dopaminérgico após a EMTr do córtex pré-frontal dorsolateral (CPFDL) (Strafella et al., 2001). Em psiquiatria, a EMTr tem sido investigada como um possível método terapêutico de modulação cortical para vários transtornos. Exemplos incluem transtorno afetivo bipolar (Dell'osso, Buoli, Hollander \& Altamura, 2010), depressão maior e transtorno obsessivo compulsivo (Ruffini et al., 2009).

Outra modalidade de estimulação cerebral não invasiva, denominada Estimulação Transcraniana por Corrente Contínua (ETCC) também ganhou relevância como uma ferramenta indolor, fácil de aplicar e capaz de modular o sistema dopaminérgico (Monte-Silva et al., 2009). Estudos revelam que a aplicação da ETCC no córtex pré-frontal - área essencial para a formulação de planos de ação e atenção seletiva - aumenta a eficiência da aprendizagem motora implícita (Kincses et al., 2004) e memória de trabalho em indivíduos saudáveis e pacientes com doença de Parkinson (Boggio et al., 2006). Outros domínios cognitivos também tem sido estudados como as funções executivas (Dockery et al., 2009) e a linguagem (Baker, Rorden \& Fridriksson, 2010).

Um grande número de estudos têm se concentrado no estudo das funções executivas no TDAH, gerando a compreensão de que os déficits executivos são uma característica central do transtorno (Miller, Loya \& Hinshaw, 2013). Os déficits cognitivos no TDAH parecem ser mais amplos não se limitando apenas aos aspectos executivos, mas abrangendo também a atenção (Hervey et al., 2004). A atenção pode ser entendida como uma complexa função cognitiva que apresenta vários subfatores. Mapou e Mateer (1996) com o objetivo de integrar vários aspectos de modelos de atenção propuseram um modelo que classifica a atenção em duas áreas: distribuição e capacidade atencional.A distribuição atencional refere-se à habilidade de focar ou modificar o foco atencional; e compreende aspectos como a vigilância, a atenção focada e sustentada. A capacidade atencional refere-se à habilidade de armazenar e manipular as informações; compreendendo a amplitude da atenção, a manipulação mental e a resistência à interferência. Tal modelo demonstra como o domínio da atenção pode ser entendido de forma complexo, multifacetado e ao mesmo também tempo integrado, de forma que sua avaliação mais apurada demanda a investigação dos vários subfatores que se interligam entre si.

Levando-se em consideração que a estimulação cerebral não invasiva atualmente abre perspectivas para o desenvolvimento de novas estratégias uteis no tratamento de indivíduos que apresentam déficits atencionais, como é o caso de pessoas com TDAH, este estudo teve como objetivo avaliar o desempenho atencional de adultos com sintomatologia de TDAH comparando-os com adultos saudáveis, após a aplicação de técnicas de estimulação cerebral não invasiva excitatória (EMTr e ETCC).

\section{Métodos}

Vinte indivíduos, de ambos os sexos, e com idade entre 19 e 50 anos participaram de um estudo crossover, controlado, randomizado e duplo-cego, realizado de acordo com protocolo aprovado pelo comitê de ética da Universidade Federal de Pernambuco. Em adição, o estudo foi registrado publicamente no site Clinical Trial Registration sob o número NCT 02250690. Todos os participantes assinaram um termo de consentimento livre e esclarecido.

O estudo foi avaliado e aprovado pelo Comitê de Ética em Pesquisa (CEP) da Universidade Federal de Pernambuco, por meio do protocolo n²53/10.

\section{Amostra do estudo e critérios de elegibilidade}

Os voluntários foram captados a partir de panfletos espalhados no Campus e no Hospital da Universidade. Os interessados em participar do estudo entraram em contato por telefones e então foram avaliados se preenchiam os critérios para a sintomatologia de TDAH. Aqueles que não preencheram os critérios puderam ser alocados no grupo controle.

Para os indivíduos com sintomatologia de TDAH, os critérios 
de inclusão utilizados foram:

- Presença de ao menos quatro itens descritos como "frequentemente" ou "muito frequentemente" em cada uma das partes da Adult Self Report Scale (ASRS-18) (Mattos et al., 2006);

- Sintomatologia intensa de desatenção e/ou hiperatividade/ impulsividade por um período de pelo menos 6 meses, de acordo com os critérios diagnósticos considerados pelo DSM-IV;

- Presença de evidências que os sintomas de desatenção ou hiperatividade/ impulsividade estiveram presentes em dois ou mais contextos (ex.: trabalho, escola de acordo com os critérios do DSM-IV;

Para os indivíduos sem sintomatologia de TDAH, os critérios de inclusão utilizados foram:

- Ausência de sintomatologia intensa de desatenção e/ou hiperatividade/ impulsividade por um período de pelo menos 6 meses, de acordo com os critérios diagnósticos considerados pelo DSM-IV;

- Ausência de sintomatologia de transtornos psiquiátricos de acordo com a Mini International Neuropsychiatric Interview (M.I.N.I) - versão 5.0.0. (Amorim, 2000);

Para ambas as populações, com ou sem sintomatologia de TDAH, os critérios de exclusão utilizados no estudo foram:

- Pacientes ou voluntários com história de epilepsia, neurocirurgia (incluindo implante de clipes metálicos), traumatismo craniano e implante de marca-passo - contraindicações formais ao uso de EMT;

- Indivíduos que fazem uso atual de drogas epileptogênicas (ex.: cocaína);

- Doença orgânica grave não controlada que possa interferir na condução do estudo, como neoplasias e cardiopatias; - Mulheres grávidas.

\section{Procedimento Experimental}

Os voluntários (com ou sem sintomatologia de TDAH) foram submetidos a duas sessões de estimulação cortical: real e fictícia (sham). A ordem das sessões foi randomizada e estas foram separadas por um período de uma semana. Os pacientes não eram cientes de qual tipo de estimulação (real ou sham) estava sendo administrada. Como no presente estudo o objetivo foi investigar o efeito da aplicação de técnicas de estimulação cerebral não invasiva do tipo excitatória no CPFDL esquerdo (CPFDLE), tanto a EMTr de alta frequência quanto a ETCC anódica foram aplicadas. A escolha da técnica ocorreu de modo aleatório entre os participantes do estudo, de modo que parte dos voluntários (com e sem sintomatologia do TDAH) fossem estimulados com a EMTr e outra parte com a ETCC.

\section{Aplicação da EMTr}

Para aplicação da EMTr, Inicialmente, os pacientes foram instruídos a sentar em uma cadeira e buscar uma posição con- fortável. Em seguida, a estimulação magnética foi aplicada através de um estimulador magnético com uma bobina em forma de 8. A bobina de estimulação foi segurada manualmente, encostada no escalpe, em um ângulo de 45 graus da linha média e apontada para a região anterior do crânio. Os seguintes parâmetros de estimulação foram utilizados:

- Local da Estimulação: A ETMr foi aplicada sobre o CPFDLE. Estudos prévios têm demonstrado que a modulação dessa área com EMTr resulta em uma melhora do funcionamento cognitivo geral do indivíduo (Moser et al., 2002). A sua marcação foi realizada correspondendo a $5 \mathrm{~cm}$ a frente da área de representação cortical do músculo primeiro interósseo dorsal.

- Intensidade da Estimulação/ Determinação do Limiar Motor de Repouso (LMR): A intensidade adotada para ETMr foi de $80 \%$ do LMR do músculo primeiro interósseo dorsal. O LMR é definido como a menor intensidade requerida do aparelho de estimulação para se obter um potencial evocado motor de pelo menos $0,05 \mathrm{mV}$ no músculo em repouso em cinco de dez tentativas (Kobayashi \& Pascual-Leone, 2003).

- Frequência e Duração das Sessões: A ETMr foi aplicada em apenas uma sessão dividida em 5 blocos. Cada bloco de estimulação foi separado por intervalos de 300s. Em cada bloco, foram aplicados 15 trens (10pulsos/trem) de EMTr com uma frequência de $10 \mathrm{~Hz}$. $\mathrm{O}$ intervalo entre as séries foi de $10 \mathrm{se}$ gundos.

A estimulação fictícia (sham) foi aplicada de modo que fosse percebida pelo paciente como o tratamento real. Assim, durante as sessões de EMTr sham foram empregados os mesmos procedimentos das sessões de EMTr real, no entanto, a estimulação foi realizada com duas bobinas: uma bobina conectada ao estimulador era colocada sobre uma cadeira próxima, mas não visível ao paciente para que o som, característico da estimulação, fosse audível, e a outra bobina, desconectada do estimulador, era posicionada sobre a cabeça do indivíduo.

\section{Aplicação da ETCC}

Para aplicação da ETCC, os voluntários foram instruídos a sentar em uma cadeira e buscar uma posição confortável. A aplicação da corrente elétrica se deu através de eletrodos de superfície. Os eletrodos utilizados em todos os experimentos desse estudo tiveram dimensões de $5 \times 7 \mathrm{~cm}(35 \mathrm{~cm} 2)$ e foram compostos por borracha condutora de eletricidade e envoltos por esponjas embebidas em soro fisiológico. Para a aplicação da ETCC, o ânodo foi posicionado sobre CPFDLE (5cm anterior à C3, sistema de marcação 10/20) e o cátodo posicionado na região supraorbital contralateral. Os parâmetros de intensidade e de duração da corrente estimulação usados foram previamente estabelecidos em humanos: intensidade de $1 \mathrm{~mA}$; duração de 20 minutos (Boggio et al., 2006).

Nas sessões de estimulações fictícias (sham) foram empregados os mesmos procedimentos das sessões de ETCC real, no entanto o aparelho de estimulação foi desligado cinco segundos após o início. 
Uma síntese do design metodológico do estudo está apresentado na Figura 1.

Figura 1 - Design metodológico do estudo 2. ASRS- 18 - Adult Self report Scale; M.I.N.I - Mini International Neuropsychiatric Interview.

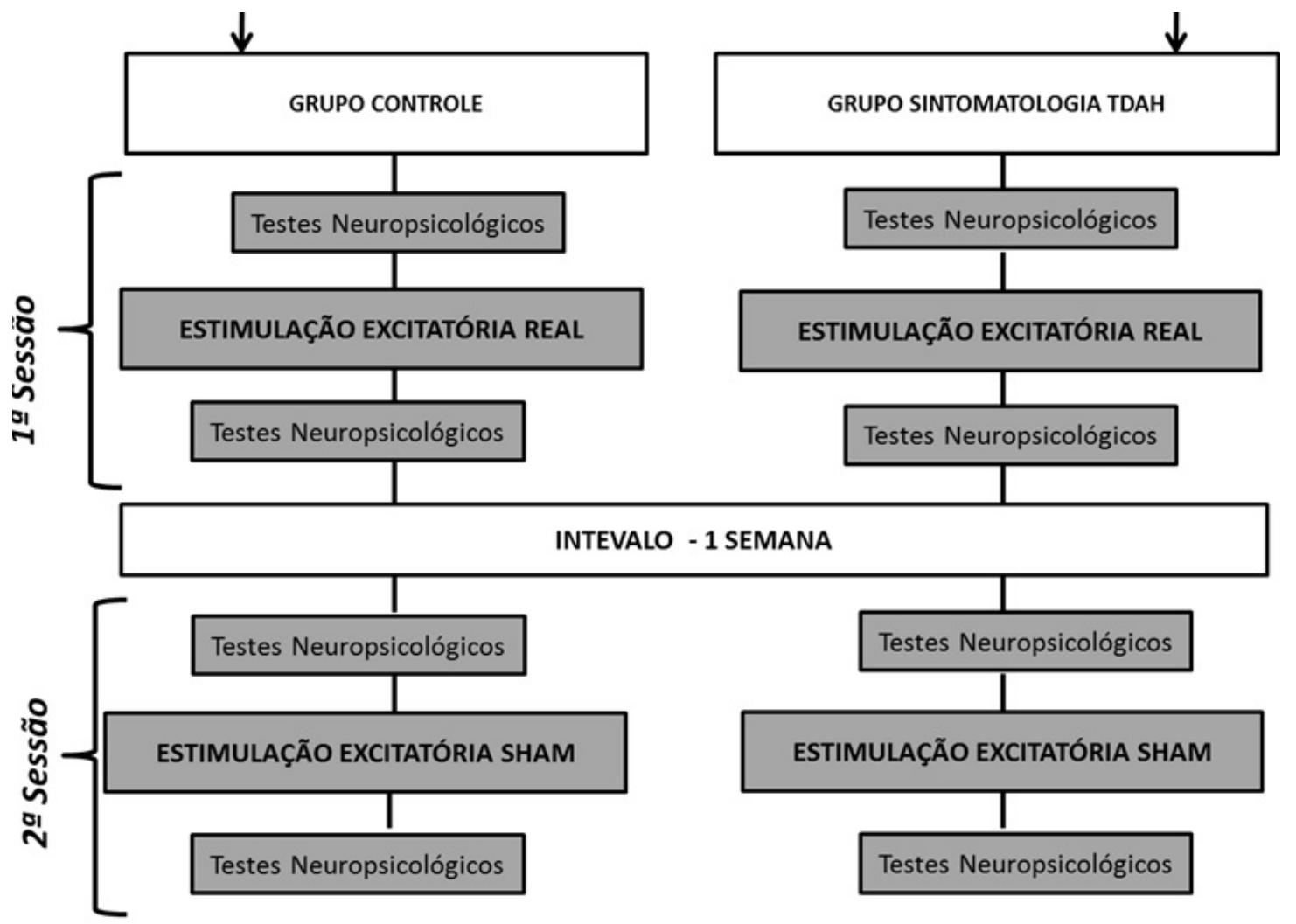

\section{Método de avaliação}

Os indivíduos foram submetidos a uma avaliação (testes neuropsicológicos) antes e imediatamente após a aplicação de estimulação cerebral não-invasiva. O pesquisador responsável pela aplicação dos testes e os voluntários desconheciam se a sessão de estimulação aplicada era real ou sham e o pesquisador responsável pela aplicação da estimulação desconhecia se o voluntário apresentava ou não sintomatologia de TDAH. Os testes usados no presente estudo foram utilizados considerando os dois eixos do modelo atencional de Mateer e Mapou (1996): capacidade e distribuição atencional.

\section{Testes neuropsicológicos utilizados para a mensuração da capacidade e distribuição atencional}

Digit span (foward/backward) - O digit span é comumente usado para mensurar a recordação verbal imediata, sendo uma medida de atenção auditiva (Lezak, 2004) e depende da capacidade de retenção a curto prazo (Shum et al., 1990). O digit span backward também tem sido usado em pesquisas como uma medida de memória de trabalho auditivo-verbal e o digit span forward, para avaliar o componente executivo central (Sjöwall \& Thorell, 2014). O digit span backward é composto por oito itens de sequências de dígitos que devem ser recordados em ordem direta após a leitura pelo examinador e o digit span forward apresenta sete itens de sequencias de números que devem ser recordados em ordem inversa. Ambos são interrompidos quando duas sequências do mesmo item são erradas. Neste estudo o teste digit span backward foi utilizado para avaliar a capacidade atencional no que se refere à amplitude.

Symbol Digit Modality Test (SDMT) -. O SDMT requer que os identifiquem nove símbolos diferentes correspondentes aos números de 1 a 9 , e preencham manualmente em espaços brancos, o número correto abaixo de cada símbolo correspondente. Na administração oral, o participante recebe uma cópia em branco do teste e pede-se que verbalize o número correto para cada símbolo correspondente. O participante possui 90 s para completar cada aplicação. A pontuação escrita e oral é calculada somando o número de respostas corretas para cada seção (Lezak et al., 2004). O SDMT é um teste utilizado para investigar a atenção dividida, velocidade visual de rastreamento e processamento motor (Strauss, Sherman \& Spreen 2006). Este estudo utilizou administração escrita do teste com o objetivo de mensurar a distribuição atencional no que se refere à atenção focada e sustentada.

Teste D2 - Caracterizado como um teste de atenção visual concentrada e no sentido mais amplo, de capacidade de concentração. É composto por 14 linhas, em que os indivíduos devem identificar as letras " $\mathrm{d}$ " que possuem dois traços, com um limite de tempo de 20 segundos para completar cada linha. Este teste requer dos examinandos diferenciação rápida e segura entre os detalhes semelhantes. O resultado do teste é avaliado pelo número total de sinais examinados - sem levar em conta se as marcações foram certas ou erradas - é 
chamado de resultado bruto (RB). Subtraindo-se o total de erros (TE), obtém-se o resultado líquido $(R L)$, fornecendo o número total de sinais corretamente analisados (Brickenkamp \& Welter, 1990). Utilizou-se o D2 para mensuração tanto de aspectos da distribuição atencional (atenção focada e sustentada) quando para mensurar a capacidade atencional no que concerne a resistência à interferência.

Trail Making Test A e B (TMT) - O TMT consiste em duas partes: A e B. Na parte A, os examinandos são requeridos a desenhar uma linha de conexão entre os círculos numerados em ordem crescente. Na parte B, a mesma tarefa é executada, no entanto, o examinando deve conectar círculos numerados alternando-os entre os números em ordem crescente e as letras em ordem alfabética (Reitan \& Wolfson, 1985). O TMT é descrito como uma medida da atenção, da velocidade de processamento e flexibilidade mental (Strauss et al., 2006). Utilizou-se o TMT- parte A para mensurar a distribuição atencional em relação à atenção focada e sustentada. O TMT-parte B foi utilizado para mensurar a capacidade atencional no que se refere à manipulação mental.

Em síntese, a esquematização dos instrumentos, considerando as áreas e subáreas atencionais segundo o modelo de Mateer e Mapou (Mapou \& Mateer, 1996), estão descritas na Tabela 1.

Tabela 1 - Organização metodológica dos testes neuropsicológicos utilizados, de acordo com o modelo atencional proposto por Mateer e Mapou (1996).

\begin{tabular}{|c|c|c|c|}
\hline Teste utilizado & Área atencional & Subárea atencional & $\begin{array}{c}\text { Parâmetro utilizado para } \\
\text { avaliação }\end{array}$ \\
\hline Digit Span Backward & Distribuição & Amplitude & Número de respostas corretas \\
\hline Digit Span Forward & Distribuição & Manipulação Mental & Número de respostas corretas \\
\hline $\begin{array}{l}\text { Digit Symbol } \\
\text { Modality Test }\end{array}$ & Capacidade & Atenção Focada e sustentada & Número de respostas corretas \\
\hline Teste D2 & $\begin{array}{l}\text { Capacidade/ } \\
\text { Distribuição }\end{array}$ & $\begin{array}{l}\text { Atenção Focada e Sustenta- } \\
\text { da/ Resistência à interferência }\end{array}$ & Resultado Líquido \\
\hline Trail Making Test - parte A & Capacidade & Atenção Focada e Sustentada & $\begin{array}{l}\text { Tempo de execução em } \\
\text { segundos }\end{array}$ \\
\hline Trai Making Test - parte B & Distribuição & Manipulação mental & $\begin{array}{l}\text { Tempo de execução em } \\
\text { segundos }\end{array}$ \\
\hline
\end{tabular}

\section{Processamento e análise dos dados}

Os dados obtidos nos testes foram processados da seguinte forma: no digit span backward/ forward foi computado o número total de sequências repetidas corretamente. Para o DSMT foram contados o número de acertos obtidos dentro do tempo de execução do teste (90 segundos). No teste D2 utilizou-se a diferença entre o número de acertos e o número de erros (resultado líquido) e no Trail Making Test (partes A e B) foi registrado em segundos, o tempo necessário para completar o teste.

Em relação à caracterização da amostra, foi realizada uma análise descritiva, usando medidas de tendência central (média e desvio-padrão) para as variáveis contínuas e medidas de frequência para as categóricas. Comparações entre os grupos com e sem sintomatologia de TDAH, com relação a variáveis de caracterização da amostra, foram realizadas utilizando-se o teste Mann-Whitney para as variáveis contínuas e o teste qui-quadrado para as variáveis categóricas.

A normalidade dos dados foi obtida a partir do teste ShapiroWilk. Na comparação intragrupos foi utilizado o teste t parea- do para as variáveis normais (Digit Symbol Modalidaty Test) e o teste de Wilcoxon para as variáveis não normais (demais testes). Na comparação entre os grupos foi utilizado o teste $t$ para amostras independentes para as variáveis normais e o teste de Mann Whitney para variáveis não normais. Em todas as análises foi utilizado o pacote estatístico SPSS versão 18.0 para Windows, com nível de significância de 0,05. 


\section{Resultados}

A Tabela 2 apresenta os dados relativos à caracterização da amostra. Comparado ao grupo controle, como esperado, observa-se que os indivíduos do grupo com sintomatologia de TDAH apresentaram maiores escores na escala ASRS-18 tanto para sintomas atencionais quanto para impulsividade/ hiperatividade, além de apresentarem outras comorbidades segundo a M.I.N.I. A partir dos resultados da ASRS-18, observa-se que os voluntários com sintomatologia de TDAH podem ser descritos como possuindo apresentação combinada (desatenção e hiperatividade/impulsividade) de acordo com os parâmetros do DSM-V.

\begin{tabular}{|c|c|c|c|}
\hline Características & $\begin{array}{l}\text { Grupo Sintomatolo- } \\
\text { gia TDAH }(n=9)\end{array}$ & $\begin{array}{l}\text { Grupo Controle } \\
\qquad(n=11)\end{array}$ & $\begin{array}{l}\text { Valor-p } \\
p<0,05\end{array}$ \\
\hline Idade $(M \pm D P)$ & $32,0 \pm 8,8$ & $23,8 \pm 4,5$ & 0,021 \\
\hline Gênero (H:M) & $4: 5$ & $5: 6$ & 0,652 \\
\hline Escore Sintomas Atencionais (ASRS-18) & $5,1 \pm 1,4$ & $1,6 \pm 0,9$ & 0,001 \\
\hline Escore Sintomas Impulsividade/Hiperatividade (ASRS-18) & $4,2 \pm 1,7$ & $1,0 \pm 1,0$ & 0,001 \\
\hline Escore Total (ASRS-18) & $9,3 \pm 3,1$ & $2,6 \pm 0,9$ & 0,001 \\
\hline Comorbidades de acordo com a M.I.N.I (M \pm DP) & $5,0 \pm 2,5$ & 0 & 0,001 \\
\hline
\end{tabular}

M = Média; DP = Desvio-Padrão; H = Homem; M = mulher;ASRS-18 = Adult Self Report Scale; M.I.N.I = Mini International Neuropsychiatric Interview. ; 1. Teste Mann Withney; 2. Teste Qui-Quadrado.

No teste digit span backward (amplitude atencional), na sessão sham, os indivíduos do grupo controle obtiveram aumento $(p=0,04)$ no número de respostas corretas (melhora do desempenho) após a estimulação fictícia, enquanto que no grupo de adultos com sintomatologia de TDAH, observou-se uma piora no desempenho $(p=0,04)$ (Figura 2$)$. No que se refere à sessão real, apesar de não sido confirmado pelo teste estatístico, a aplicação da estimulação cerebral parece interferir positivamente, promovendo uma melhora do desempenho dos indivíduos com sintomatologia de TDAH. No entanto, um efeito oposto (prejuízo na amplitude atencional) parece ocorrer no grupo controle após a estimulação.

Figura 2 - Desempenho dos grupos controle e com sintomatologia de TDAH no Digit Spanbackward. As colunasrepresentam o número de respostas corretas obtido pela diferença obtida entre os escores do teste pós-estimulação e pré-estimulação. As barras de erros representam o erro-padrão da média e os asteriscos indicam diferença significativa em relação ao resultado pré-estimulação

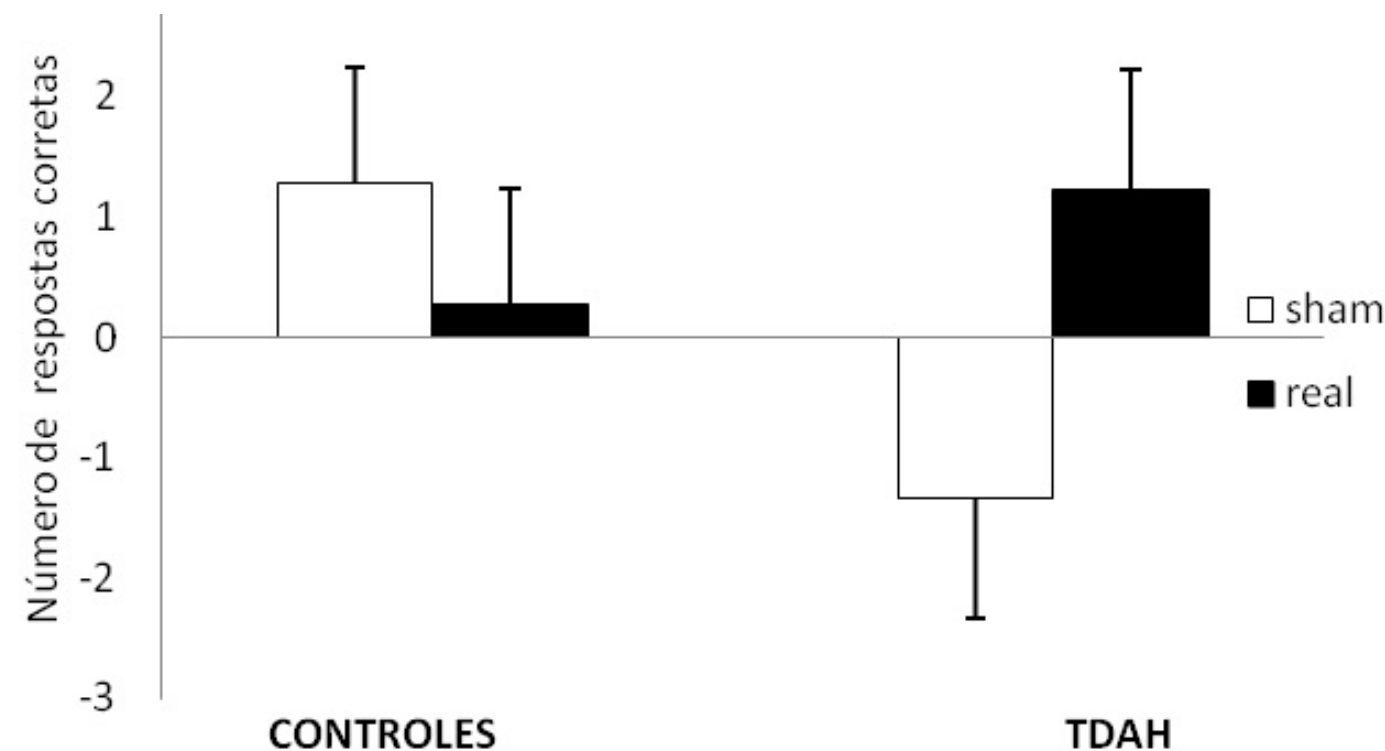


Um padrão semelhante ao resultado do digit span backward parece ocorrer no teste digit span forward(Figura 3), em que há uma redução no número de respostas corretas quando os indivíduos do grupo controle são submetidos à estimulação real e padrão inverso no grupo com sintomatologia de TDAH. No entanto, não foi verificada diferença estatística nas comparações intra e intergrupos.

Figura 3 - Desempenho dos grupos controle e com sintomatologia de TDAH no Digit Spanforward. As colunasrepresentam o número de respostas corretas obtido pela diferença obtida entre os escores do teste pós-estimulação e pré-estimulação. As barras de erros representam o erro-padrão da média.

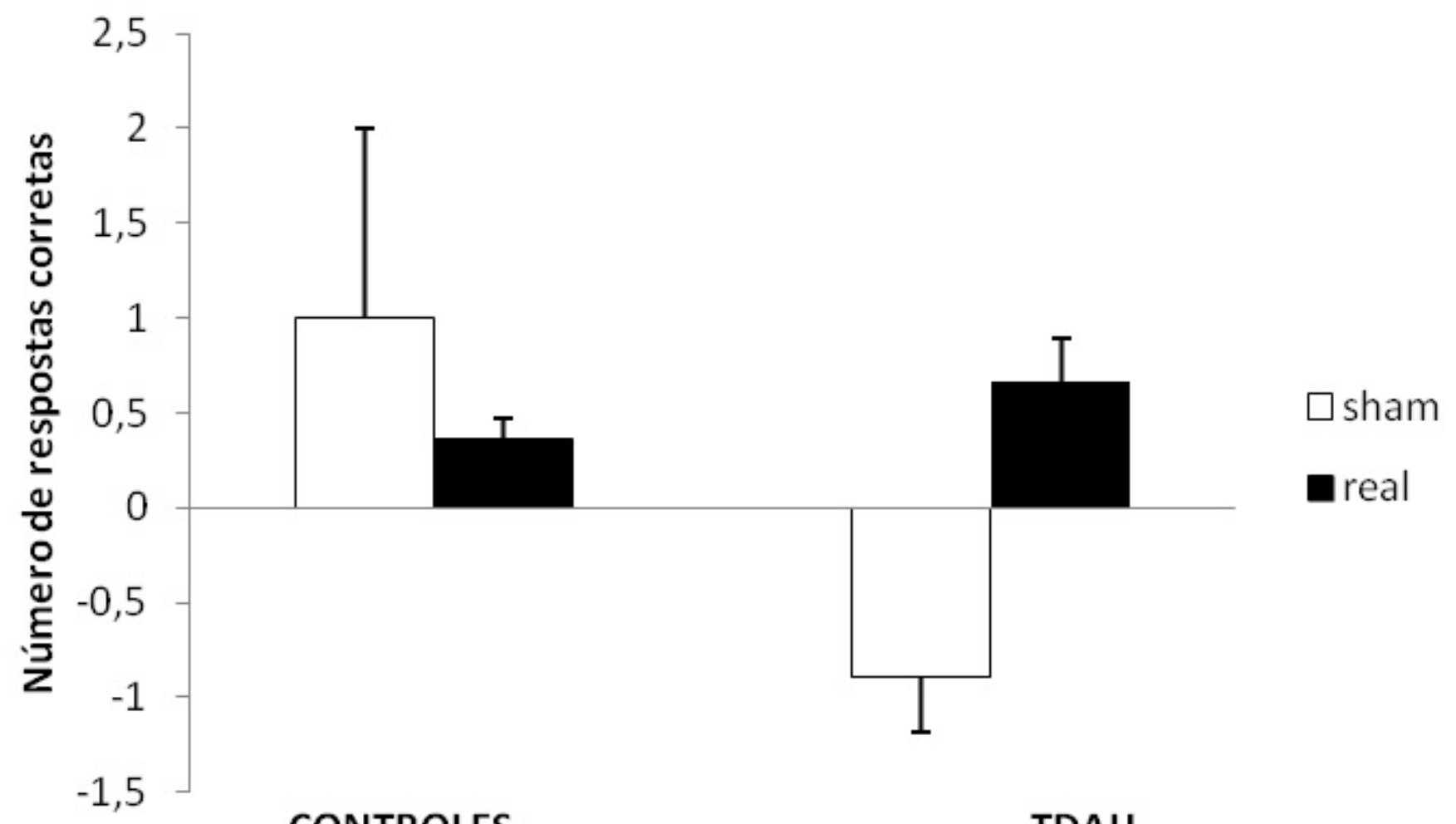

\section{CONTROLES}

TDAH

No que concerne à mensuração da atenção focada e sustentada através do Digit symbol modality test (Figura 4), também não foi verificada diferença estatística nas comparações intra e intergrupos. No entanto, uma melhora no desempenho da atenção focada e sustentada, revelada através do aumento do número de acertos no teste, parece ocorrer no grupo com sintomatologia de TDAH após a estimulação real. Esse efeito positivo da estimulação sobre a atenção focada e sustentada foi confirmada pelo Trail Making Test (parte A; Figura 5), no qual, foi observado uma redução $(p=0,01)$ do tempo de execução do teste, indicativo de melhora no desempenho atencional, quando os indivíduos com sintomatologia de TDAH foram submetidos a estimulação cerebral sobre o CPFDLE. Similar aos outros testes, no Trail Making Test (parte A), a estimulação real parece de alguma forma prejudicar o desempenho atencional dos voluntários do grupo sem sintomatologia de TDAH (grupo controle). 
Figura 4 - Desempenho dos grupos controle e com sintomatologia de TDAH Digit Symbol Modality test. As colunas representam o número de respostas corretas obtido pela diferença obtida entre os escores do teste pós-estimulação e pré-estimulação. As barras de erros representam o erro-padrão da média

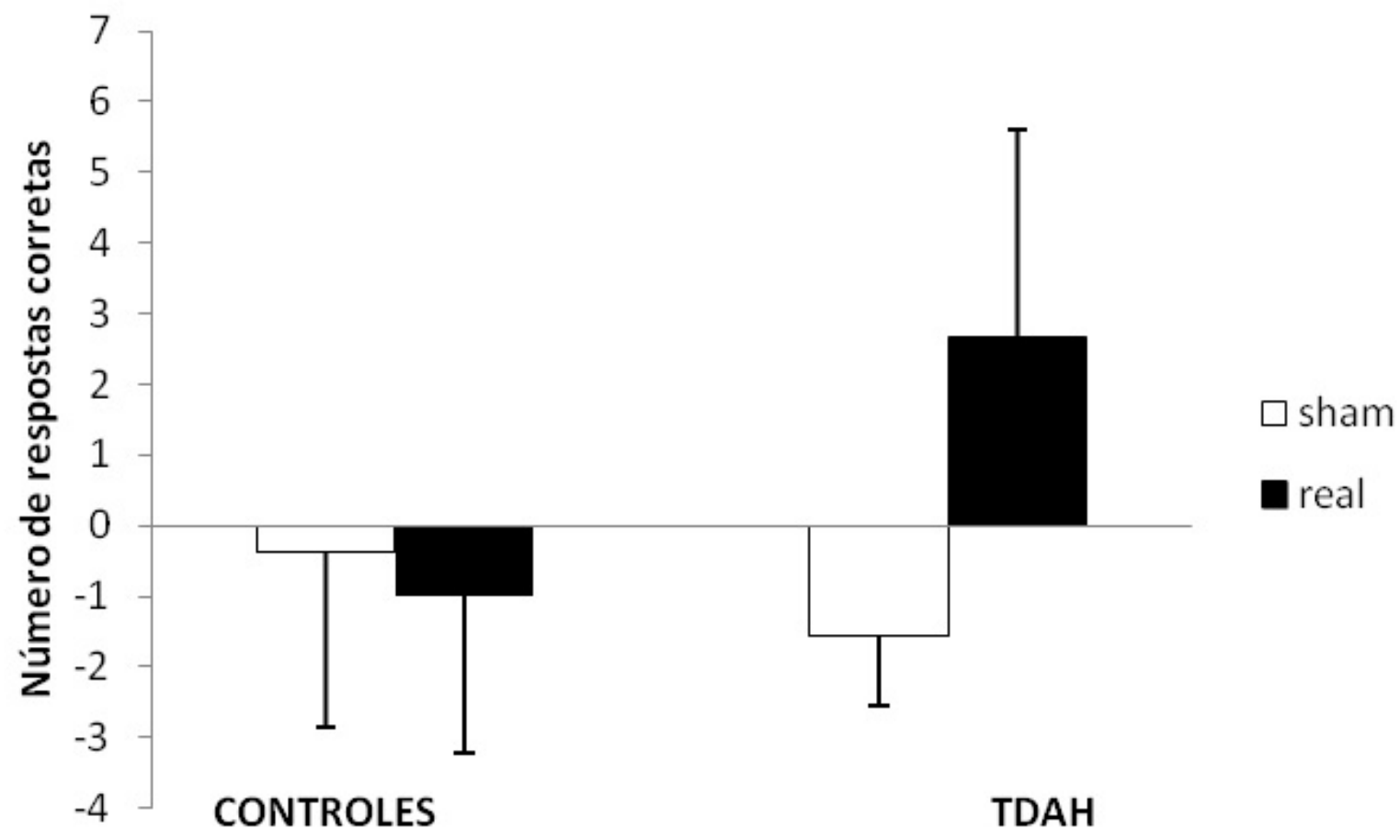

Figura 5 - Desempenho atencional, calculado a partir do tempo de execução em segundos, dos grupos controle e com sintomatologia de TDAH no Trail Making Test - parte A. As colunasrepresentama diferença de tempo de execução do teste pós-estimulação com o da pré-estimulação. As barras de erros representam o erro-padrão da média e os asteriscos indicam diferença significativa em relação ao resultado pré-estimulação.

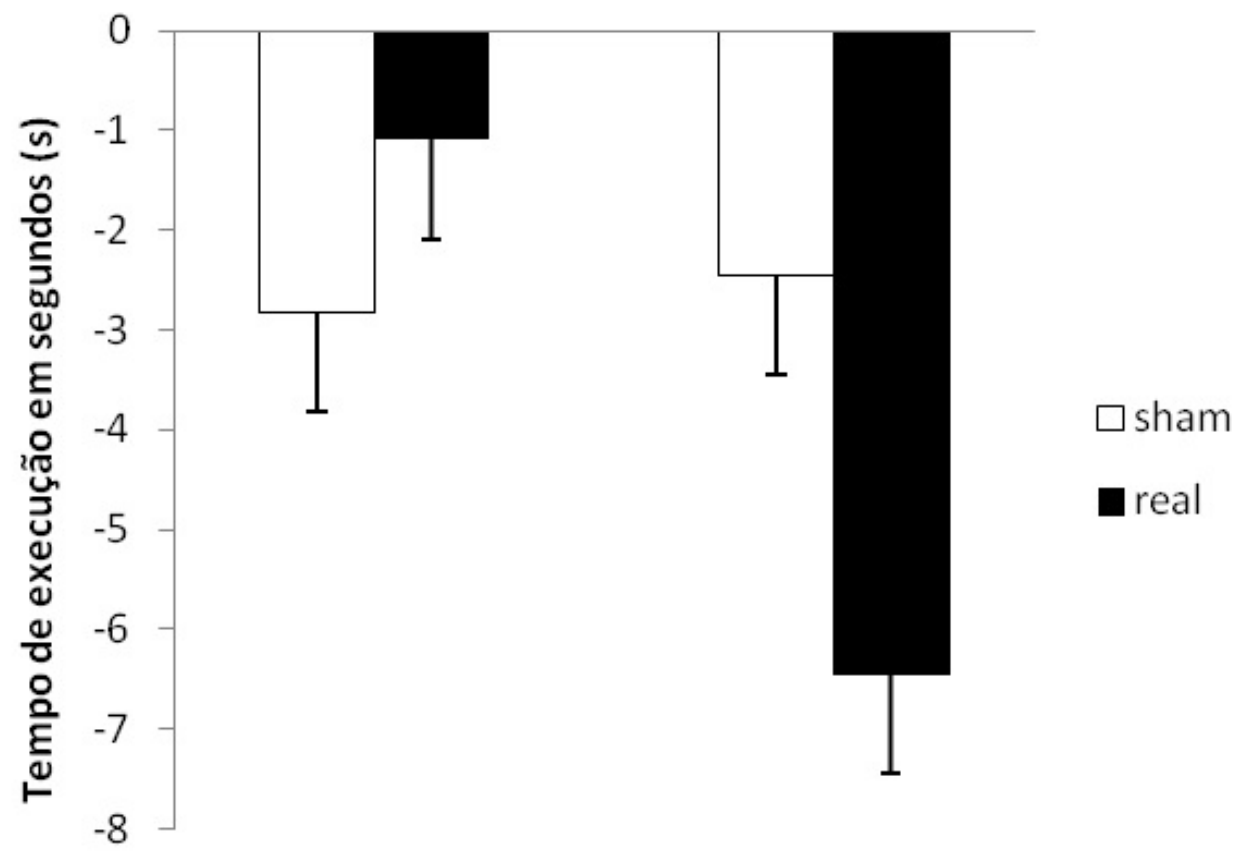


Similarmente, no D2 test, que além da atenção focada e sustentada analisa também a capacidade atencional do indivíduo com relação à resistência à interferência, também foi observado o benefício (aumento do número de acertos) da aplicação da estimulação cerebral no grupo com sintomatologia de TDAH (Figura 6). No grupo controle, quando comparado com a estimulação sham, a estimulação real parece não interferir no desempenho do teste.

Figura 6 - Desempenho dos grupos controle e com sintomatologia de TDAH no teste D2. As colunasrepresentam a variação do resultado líquido (diferença entre o número de acertos e o número de erros) do teste pós-estimulação com relação ao resultado líquido pré-estimulação. As barras de erros representam o erro-padrão da média e os asteriscos indicam diferença significativa em relação ao valores pré-estimulação

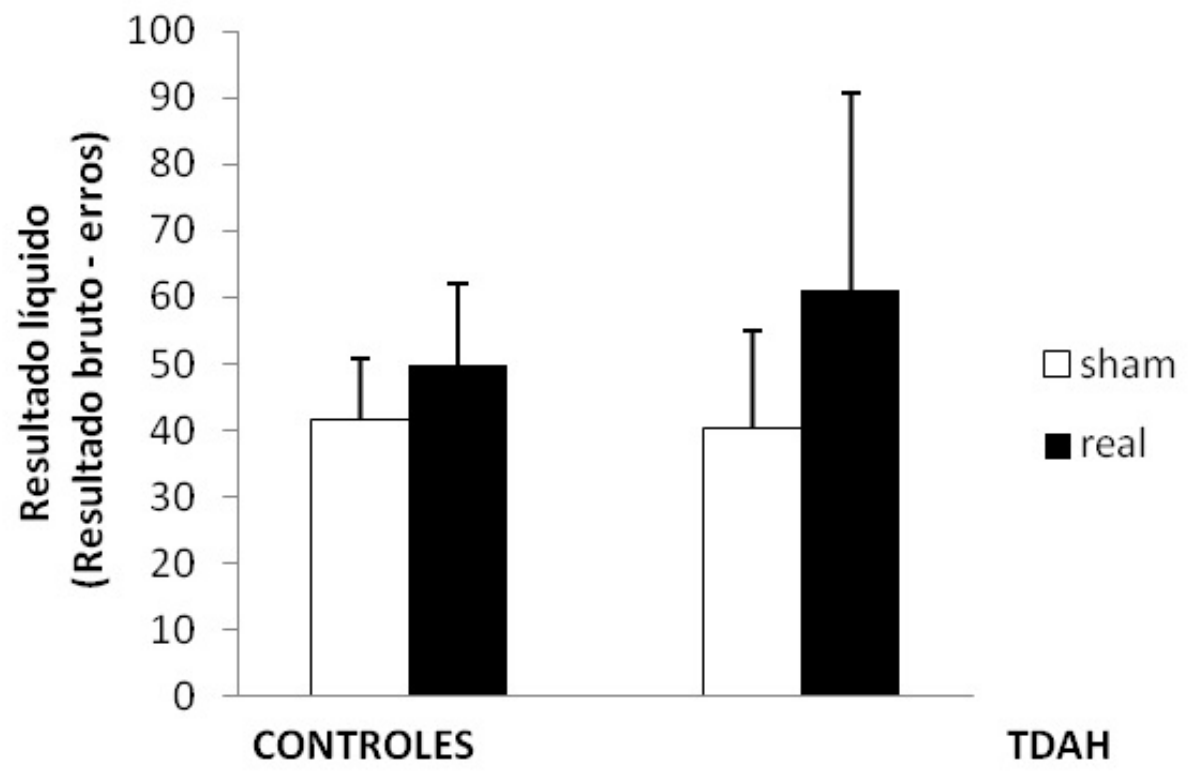

No Trail Making Test (parte B), que avalia a manipulação mental, a estimulação real aprece prejudicar o desempenho atencional em ambos os grupos (Figura 7).

Figura 7 - Desempenho dos grupos controle e com sintomatologia de TDAH no Trail Making Test - parte B. As colunasrepresentama diferença de tempo de execução do teste pós-estimulaçãocom o da pré-estimulação. As barras de erros representam o erro-padrão da média e os asteriscos indicam diferença significativa em relação ao tempo de execução pré-estimulação.

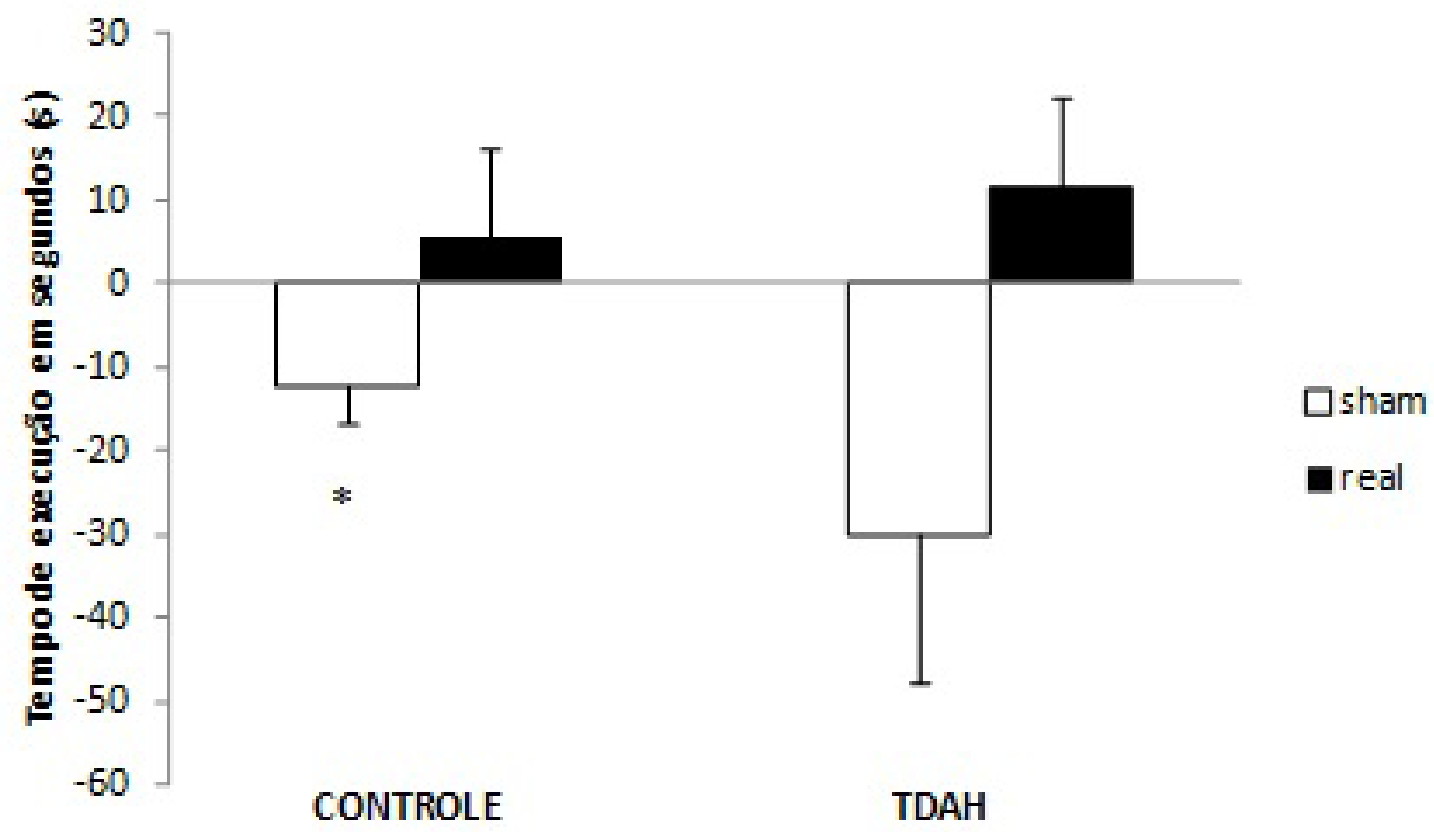




\section{Discussão}

Em suma, os resultados obtidos neste estudo corroboram a hipótese de que a estimulação cerebral não invasiva excitatória aplicada sobre o CPFDLE pode aumentar o desempenho atencional de adultos com sintomatologia de TDAH, mais especificamente em relação à amplitude atencional, a atenção focada e sustentada e resistência à interferência. No entanto, para os adultos saudáveis (sem sintomatologia de TDAH), a estimulação cerebral parece prejudicar o desempenho em testes atencionais.

Em relação ao uso das técnicas de estimulação cerebral não invasiva, até onde se tem conhecimento, apenas dois estudos utilizaram a EMT com proposta terapêutica em indivíduos com TDAH (Bloch et al., 2010). Niederhofer (2008) apresenta um estudo de caso, em que se aplicou EMTr de baixa frequência $(1 \mathrm{~Hz})$ na área motora suplementar pelo período de 5 dias e com duração de uma hora em uma mulher com 42 anos com diagnóstico de TDAH. Após as sessões, a sintomatologia do transtorno mensurada através da Conner's Rate for Adults pareceu reduzir discretamente. No estudo de Bloch et al. (2010), a EMTr de alta frequência $(20 \mathrm{~Hz})$ foi aplicada sobre o córtex pré-frontal dorsolateral direito, em uma única sessão, em 24 indivíduos com TDAH. Uma melhora atencional avaliada através do questionário The positive and Negative Affect Schedule foi observada após estimulação.

Em nosso estudo, observou-se que sem a estimulação cerebral (sessão sham) os adultos sem sintomatologia de TDAH (grupo controle) aumentaram o desempenho atencional, tornando-se mais hábeis em estabelecer e manter o foco quando observado nos testes Digit Span Backward, Trail Making (parte A) e D2, o que não aconteceu com adultos com sintomas de TDAH. Uma possível explicação para esse resultado é que o aumento no desempenho dos testes no grupo controle pode ter se dado a partir do efeito de aprendizagem, considerando a segunda exposição ao teste (avaliação pós- estimulação fictícia). Se esta hipótese for considerada, pode-se concluir que o efeito aprendizagem não esteve presente no grupo de indivíduos com sintomatologia de TDAH. Ao contrário, a segunda exposição aos testes pode prejudicar seu desempenho quando a estimulação cerebral não for aplicada, como ocorreu, por exemplo, no teste Digit Span Backward.

A aprendizagem pode ser definida como o processo de aquisição de informações, resultando na formação de uma memória. Um estudo recente que investigou os processos de memória em 97 homens com TDAH e dificuldades de aprendizagem, observou que estes na sua maioria mostraram prejuízos nos domínios de memória de longo prazo e, parcialmente, na memória de curto prazo, sugerindo que a dificuldade de aprendizagem interfere nos mecanismos de consolidação.

O efeito de aprendizagem nos testes (digit span backward, Trail Making-parte A-e D2) parece ocorrer nos indivíduos com sintomatologia de TDAH apenas quando a estimulação excitatória real foi aplicada no CPFDLE, sinalizando que a estimulação induziu a uma melhora no desempenho atencional dos indivíduos quando relacionado a atenção focada e sustentada e na habilidade de resistência à interferência. Estudo prévio em indivíduos com Parkinson também tem demonstrado o benefício do uso da aplicação da estimulação cerebral do tipo excitatória (ETCC anódica) aplica no CPFDLE sobre a memória operacional dos pacientes (Boggio et al., 2006).

Os mecanismos envolvidos neste efeito positivo da estimulação sobre o desempenho atencional de indivíduos que não se beneficiam com o efeito de aprendizagem ainda precisa ser melhor elucidado. No entanto, pode-se especular que a estimulação cerebral tenha ocasionado um aumento da concentração de dopamina no córtex pré-frontal e isso ter refletido na melhora atencional dos voluntários com sintomatologia de TDAH em nosso estudo. Essa hipótese é baseada em estudos que apontam que no TDAH, o que parece estar alterado no córtex pré-frontal é o funcionamento do sistema de neurotransmissores, principalmente da dopamina e que é possível modular o sistema DOPAminérgico através da estimulação cerebral não invasiva do córtex dorsolateral pré-frontal (Strafella et al., 2001). Mais estudos são necessários para confirmar os mecanismos envolvidos nos efeitos da estimulação sobre o desempenho atencional de indivíduos com TDAH.

Ainda em relação aos resultados do nosso estudo outro aspecto merece destaque. Em alguns testes (Digit Span Backward e o Trail Making - parte B), a estimulação cerebral excitatória parece induzir a uma diminuição do efeito aprendizagem observado nos adultos sem sintomatologia de TDAH, resultado aposto ao observado nos indivíduos com sintomatologia. Tal observação parece indicar que, de alguma forma, o funcionamento cortical habitual e sua estabilidade foi prejudicado quando os adultos sem TDAH foram expostos à estimulação.

No que concerne o uso da estimulação cerebral não invasiva excitatória em indivíduos saudáveis, existem dados que corroboram este achado. Rami et al. (2003), por exemplo, aplicaram em 16 homens saudáveis EMTr de alta frequência no CPFDLE e observaram rebaixamento no desempenho nas tarefas de memória episódica. Ainda em relação a sujeitos saudáveis submetidos à intervenção objetivando modulação cerebral, Pineda et al. (Pineda et al., 2008) realizaram estudo com 13 crianças portadoras de autismo comparando-as com crianças saudáveis, submetendo-as a 30 horas de aplicação de neurofeedback e avaliando-as pré e pós neuromodulação. Dentre os resultados, verificou-se que as crianças saudáveis pioraram nos escores do Autismo Treatment evaluation checklist (ATEC) e do Vineland Adaptative Behaviour Scales (VinelandII); instrumentos que observam várias características comportamentais relacionadas ao transtorno. Apesar de tratar-se de intervenção e transtorno distintos, tal achado, assim como nosso estudo, observou que o benefício da neuromodulação cortical limitou-se ao grupo de pacientes, afetando os grupos diferentemente. Hipoteticamente, parece haver mecanismo neurofisiológico específico capaz de provocar resultados distintos entre grupos de pacientes e de sujeitos saudáveis. Nesta ótica, no nosso estudo, o funcionamento cortical dos indivíduos saudáveis parece desequilibrar-se momentaneamente, enquanto aqueles com sintomatologia de TDAH equilibrar-se em habilidades esperadas em funcionamento habitual.

É importante ressaltar que os resultados obtidos neste estudo precisam ser interpretados com cautela, por certas limitações. Primeiro, a amostra do estudo foi pequena, uma vez que a população escolhida para o estudo ainda é de difícil captação. É possível que este tenha sido o motivo da falta de confirmação do teste estatístico dos resultados dos testes Digit span forward e no Digit symbol modality. Segundo, apesar dos procedimentos de estimulação utilizados serem consonantes 
nas duas técnicas aplicadas (EMTr e ETCC), tendo como fim a modulação através do aumento da excitabilidade cortical; e o objetivo do estudo ter sido avaliar o efeito da estimulação excitatória não-invasiva na população já mencionada, é possível que as técnicas de estimulação possam ter efeitos distintos sobre o desempenho atencional dos pacientes com TDAH. Assim, sugere-se que estudos futuros possam, a partir de composição de amostra maior, estratificar os resultados em relação a cada técnica isoladamente.

Até onde se tem conhecimento, não há estudos que empregaram apenas tDCS anódica sobre o desempenho atencional de adultos com TDAH. Este estudo também se limitou a identificar tais efeitos a partir de uma única aplicação de estimulação cerebral, da mesma forma que o já citado estudo de Bloch et al. (2010). Futuros estudos podem elaborar um plano de tratamento composto por várias sessões, inclusive com a realização de follow-up para a avaliação da duração de efeitos.

Também é importante ressaltar que os testes utilizados neste estudo, podem ser vistos como instrumentos convencionais, construídos dentro de um paradigma não ecológico. Testes ecológicos são formulados para imitar situações do mundo real e têm sido de interesse crescente na neuropsicologia (Torralva et al., 2013). Em estudo recente, Torralva et al. (2013) observaram que mediante o uso de testes neuropsicológicos convencionais, adultos com TDAH que apresentavam um perfil neuropsicológico de alto funcionamento - mensurado através de uma bateria padrão - não apresentaram resultados diferentes do grupo controle. Entretanto, quando submetidos à tarefa ecológica e testes computadorizados específicos, observou-se diferença significativa entre os grupos. Dessa forma, testes padrão podem apresentar baixa sensibilidade para discriminar indivíduos saudáveis de portadores de TDAH. Destaca-se que estudos ainda são carentes de metodologias que contemplem avaliações neuropsicológicas mais próximas ao funcionamento cotidiano e uniformização metodológica que permitam comparação de resultados.

Os achados do presente estudo ratificam a compreensão que a estimulação cerebral não invasiva excitatória pode contribuir para melhora cognitiva em adultos acometidos por sintomas do déficit de atenção e hiperatividade, e sugerem que processo inverso pode acontecer em relação aos indivíduos saudáveis.

\section{Referencias}

Amorim, P. (2000). Mini International Neuropsychiatric Interview (MINI): validação de entrevista breve para diagnóstico de transtornos mentais. Revista Brasileira de Psiquiatria. 22(3), 106-115. https://dx.doi.org/10.1590/ S1516-44462000000300003

Baker, J.M, Rorden, C. \& Fridriksson, J. (2010) Using transcranial direct-current stimulation to treat stroke patients with aphasia. Stroke, 41(6). 1229-1236. doi: 10.1161/ STROKEAHA.109.576785

Benczik, E. B., (2000) Manual da escala de transtorno de déficit de atenção/hiperatividade: versão para professores. São Paulo: Casa do Psicólogo,

Bloch, Y., Harel, EV., Aviram, S., Govezensky, J., Ratzoni, G. \& Levkovitz, Y. (2010) Positive effects of repetitive transcranial magnetic stimulation on attention in ADHD Subjects: a randomized controlled pilot study. The World Journal of Biological Psychiatry. 11(5). 755758. doi: 10.3109/15622975.2010.484466.

Boggio, P., Ferrucci, R., Rigonatti, S., Covre, P., Nitsche, M., Pascual-Leone, A. \& Fregni, F. (2006). Effects of transcranial direct current stimulation on working memory in patients with Parkinson's disease. Journal of the neurological sciences, 249(1). 31-38. doi: 10.1016/j.jns.2006.05.062

Brickenkamp, R .\& Welter, G. M. R. (1990) Teste d2: atenção concentrada: manual, instruções, avaliação, interpretação. Casa do Psicólogo.

Bush, G., Frazier, J. A., Rauch, S. L., Seidman, L. J., Whalen, P. J., Jenike, M. A., . . Biederman, J. (1999). Anterior cingulate cortex dysfunction in attention-deficit/hyperactivity disorder revealed by $f M R I$ and the Counting Stroop. Biological Psychiatry, 45(12), 1542-1552. Doi: 10.1016/S0006-3223(99)00083-9

Casey, B., Castellanos, F.X., Giedd, J.N., Marsh, W.L., Hamburger, S.D., et al. (1997) Implication of right frontostriatal circuitry in response inhibition and attention-deficit/ hyperactivity disorder. Journal of the American Academy of Child \& Adolescent Psychiatry, 36(3), 374383. Doi: $10.1097 / 00004583-199703000-00016$

Dell'Osso, B., Buoli, M., Hollander, E. \& Altamura AC. (2010) Duration of untreated illness as a predictor of treatment response and remission in obsessive-compulsive disorder. The World Journal of Biological Psychiatry. 11(1),59-65. doi: 10.3109/15622970903418544

Dockery, C., Hueckel-Weng, R., Birbaumer, N. \& Plewnia, C. (2009) Enhancement of planning ability by transcranial direct current stimulation. The Journal of Neuroscience, 29(22) 7271-7277. doi: https://doi.org/10.1523/ JNEUROSCI.0065-09.2009

Ernst, M., Zametkin, A.J., Matochik, J.A., Pascualvaca, D., Jons, P.H., \& Cohen, R.M. (1999) High midbrain [18F] DOPA accumulation in children with attention deficit hy- 
peractivity disorder. American Journal of Psychiatry. 156,(8). 1209-1215.

Faraone, S., Spencer, T., Aleardi, M., Pagano, C. \& Biederman, J. (2004) Meta-analysis of the efficacy of methylphenidate for treating adult attention-deficit/hyperactivity disorder. Journal of clinical psychopharmacology. 24(1), 24-29. doi: 10.1097/01.jcp.0000108984.11879.95

Giedd JN, Castellanos FX, Casey BJ, Kozuch P, King AC, Hamburger SD, Rapoport JL. (1994), Quantitative morphology of the corpus callosum in attention deficit hyperactivity disorder. The American Journal of Psychiatry. 151-665-669. doi: 10.1176/ajp.151.5.665

Hervey, A. S., Epstein, J. N. \& Curry, J. F. (2004) Neuropsychology of adults with attention-deficit/hyperactivity disorder: a meta-analytic review. Neuropsychology, 18(3), 485-503. doi:10.1037/0894-4105.18.3.485

Kincses, T., Antal, A., Nitsche, M., Bártfai, O \& Paulus, W. (2004) Facilitation of probabilistic classification learning by transcranial direct current stimulation of the prefrontal cortex in the human. Neuropsychologia. 42(1)113-117.

Kobayashi, M. \& Pascual-Leone, A.(2003). Transcranial magnetic stimulation in neurology. The Lancet Neurology. 2(3). 145-156.

Lezak, M., Howieson, D., Bigler, E. \& Tranel, D. (2004). Neuropsychological assessment. Oxford University Press, USA.

Mapou, R. L. \& Mateer, C. A. (1996) Understanding, evaluating, and managing attention disorders following traumatic brain injury. The Journal of Head Trauma Rehabilitation. 11(2), 1-16.

Mattos, P., Segenreich, D., Saboya, E., Louzã, M., Dias, G, \& Romano, M. (2006). Adaptação transcultural para o português da escala Adult Self-Report Scale para avaliação do transtorno de déficit de atenção/hiperatividade (TDAH) em adultos. Archives of Clinical Psychiatry (São Paulo), 33(4), 188-194. https://dx.doi. org/10.1590/S0101-60832006000400004

Miller, M., Loya, F. \& Hinshaw, S. P. (2013) Executive functions in girls with and without childhood ADHD: developmental trajectories and associations with symptom change. Journal of Child Psychology and Psychiatry. 54(9). 1005-1015. doi: 10.1111/jcpp.12074

Missonnier, P., Hasler, R., Perroud, N., Herrmann, F. R., Millet, P., et al. (2013) EEG anomalies in adult ADHD subjects performing a working memory task. Neuroscience, $v$. 241, p. 135-146. doi: 10.1016/j.neuroscience.2013.03.011

Monte-Silva, K., Kuo, MF., Thirugnanasambandam, N., Liebetanz, D., Paulus, W. \& Nitsche, MA. (2009) Dose-dependent inverted U-shaped effect of dopamine (D2-like) receptor activation on focal and nonfocal plasticity in humans. The Journal of Neuroscience 29(19). 6124-6131. doi: 10.1523/JNEUROSCl.0728-09.2009.

Moser, D., Jorge, R., Manes, F., Paradiso, S., Benjamin, M. \&
Robinson, R. (2002) Improved executive functioning following repetitive transcranial magnetic stimulation. Neurology. 58(8)1288-1290.

Niederhofer, H. (2008) Effectiveness of the repetitive Transcranical Magnetic Stimulation (rTMS) of $1 \mathrm{~Hz}$ for Attention-Deficit Hyperactivity Disorder (ADHD). Psychiatria Danubina. 20(1) 91-92.

Pineda, J.A., Brang, D., Hecht, E., Edwards, L., Carey, S., Bacon, M., Fitagaki, C., Suck, D., Tom, J., Birnbaum, C. \& Rork, A. (2008) et al. Positive behavioral and electrophysiological changes following neurofeedback training in children with autism. Research in Autism Spectrum Disorders. 2(3). 557-581.

Rami, L., Gironell, A., Kulisevsky, J., García-Sánchez, C., Berthier, M. \& Estévez-González, A. (2003) Effects of repetitive transcranial magnetic stimulation on memory subtypes: a controlled study. Neuropsychologia, 41(14). 1877-1883.

Reitan, R. M. \& Wolfson, D. (1985) The Halstead-Reitan neuropsychological test battery: Theory and clinical interpretation. Reitan Neuropsychology.

Ruffini, C., Locatelli, M., Lucca, A., Benedetti, F., Insacco, C. \& Smeraldi, E. (2009) Augmentation effect of repetitive transcranial magnetic stimulation over the orbitofrontal cortex in drug-resistant obsessive-compulsive disorder patients: a controlled investigation. Primary care companion to the Journal of clinical psychiatry. 11(5). 226-230. doi: 10.4088/PCC.08m00663

Shum, D. H., Mcfarland, K. A. \& Bain, J. D. (1990) Construct validity of eight tests of attention: Comparison of normal and closed head injured samples. The Clinical Neuropsychologist 4(2). 151-162.

Sjöwall, D. \& Thorell, L. B. (2014) Functional Impairments in Attention Deficit Hyperactivity Disorder: The Mediating Role of Neuropsychological Functioning. Developmental neuropsychology. 39(3) 187-204. doi: $10.1080 / 87565641.2014 .886691$

Strafella AP, Paus T, Barrett J. \& Dagher A. (2001). Repetitive transcranial magnetic stimulation of the human prefrontal cortex induces dopamine release in the caudate nucleus. The Journal of Neuroscience. 21(15). 1-4.

Strauss, E., Sherman, E. M. \& Spreen, O. (2006) A compendium of neuropsychological tests: Administration, norms, and commentary. Oxford University Press, USA.

Torralva, T., Gleichgerrcht, E., Lischinsky, A., Roca, M. \& Manes, F. (2013) "Ecological" and highly demanding executive tasks detect real-life deficits in high-functioning adult ADHD patients. Journal of attention disorders. 17(1). 11-19. doi: 10.1177/1087054710389988.

Woltering, S., Liu, Z.-X., Rokeach, A., Tannock, R. (2013). Neurophysiological differences in inhibitory control between adults with ADHD and their peers. Neuropsychologia, 51(10), 1888 - 1895. doi:10.1016/j.neuropsychologia.2013.06.023 